gap> $\mathrm{g}:=$ SymmetricGroup ( 4 );

$\operatorname{Sym}([1 \ldots 4])$

i5 : betti $(t$, Weights $\Rightarrow\{1$, gap $\}$

false

01234 gap> tblmod2:= CharacterTable( tbl, 2);

05 = total: 1413144 BrauerTable $(\operatorname{Sym}([1 \ldots 4]), 2$ )

1: . 2242 gap> tblmod2 = CharacterTable $(t b l, 2)$;

Journal of Software for

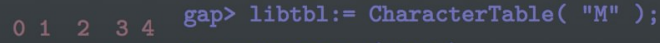

$06=$ total: 1413144 CharacterTable( "M")

fail $\quad r i n g ~ r 1=32003,(x, y, z)$, ds ;

gap> CharacterTable ( "Symmetric", 4 ) ; int a, b, c, t=11,5,3,0;

BettiTally

CharacterTable ( "Sym(4)" )

gap> ComputedBrauerTables ( tbl );

$\begin{array}{llrrr}0 & 1 & 2 & 3 & 4\end{array}$

poly $f=x^{\wedge} a+y^{\wedge} b+z^{\wedge}(3 * c)+x^{\wedge}(c+2) * y^{\wedge}(c-1)+x^{\wedge}$ $x^{\sim}(c-2) * y^{\wedge} c *\left(y^{\sim} 2+t * x\right)^{\wedge} 2 ;$

o7 = total: 1413144

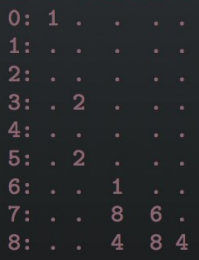

o7 : BettiTally

i8 : peek t1

$08=\operatorname{BettiTally}\{(0,\{0,0\}, 0) \Rightarrow 1\}$

$(1,\{2,2\}, 4) \Rightarrow 2$

$(1,\{3,3\}, 6) \Rightarrow 2$

(2, $\{3,7\}, 10) \Rightarrow 2$

$(2,\{4,4\}, 8) \Rightarrow 1$

(2, $\{4,5\}, 9) \Rightarrow 4$

$(2,\{5,4\}, 9) \Rightarrow 4$

2,4 terior Ideals: a package for computing

$(3,\{5$ momomial ideals in an exterior algebra

$(3,\{7,4\}, 11) \Rightarrow 2$

$(4,\{7,5\}$, LUUe AMATA AND MARILENA CRUPI

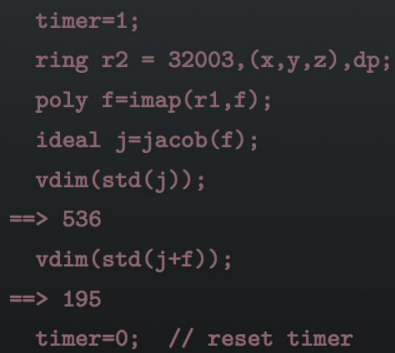




\title{
ExteriorIdeals: a package for computing monomial ideals in an exterior algebra
}

\author{
LuCA AmATA AND MARILENA CRUPI
}

\begin{abstract}
Let $K$ be a field, $V$ a $K$-vector space with basis $e_{1}, \ldots, e_{n}$, and $E$ the exterior algebra of $V$. We introduce a Macaulay 2 package that allows one to deal with classes of monomial ideals in $E$. More precisely, we implement in Macaulay 2 some algorithms in order to easily compute stable, strongly stable and lexsegment ideals in $E$. Moreover, an algorithm to check whether an $(n+1)$-tuple $\left(1, h_{1}, \ldots, h_{n}\right)\left(h_{1} \leq n=\operatorname{dim}_{K} V\right)$ of nonnegative integers is the Hilbert function of a graded $K$-algebra of the form $E / I$, with $I$ a graded ideal of $E$, is given. In particular, if $H_{E / I}$ is the Hilbert function of a graded $K$-algebra $E / I$, the package is able to construct the unique lexsegment ideal $I^{\text {lex }}$ such that $H_{E / I}=H_{E / I^{\text {lex }}}$.
\end{abstract}

1. Introduction. Monomial ideals are a bridge between algebra and combinatorial algebra. It is well known that, even if such ideals are, in some sense, among the simplest structures in commutative algebra, they are the main objects of combinatorial commutative algebra. Many authors have focused their attention on classes of monomials ideals in an exterior algebra [Aramova et al. 1997; 2000; Crupi and Utano 1999; 2007; Crupi and Ferró 2015; Eisenbud et al. 2003; Crupi 2015; Gasharov 1997; Murai 2011; Shakin 2004; 2005] and on the behavior of certain invariants, such as for instance, the Hilbert function.

In this paper, we introduce ExteriorIdeals.m2 - a new package written for [Macaulay2] for manipulating special classes of monomial ideals in an exterior algebra of a finite-dimensional vector space over a field. More precisely, the package provides functions to check whether a monomial ideal is stable, strongly stable, or lexsegment, and, respectively, to compute the smallest stable, strongly stable, or lexsegment ideal containing a given monomial ideal. Moreover, given an exterior algebra, the package allows the computation of all the Hilbert sequences of quotients of the exterior algebra. Some utility functions are necessary to simplify and optimize the implementation of the main algorithms, such as the Macaulay expansion, the initial degree of a graded ideal, the support of a monomial and the shadow of a set of monomials. Most of the algorithms must work in an exterior algebra

MSC2010: 13A02, 15A75, 68W30.

Keywords: exterior algebra, monomial ideals, Hilbert functions, algorithms.

ExteriorIdeals.m2 version 1.0 
endowed with the lexicographic order, so that such an ordering is forced within routines. Nevertheless, the ideals obtained by our algorithms are made compatible with the native exterior algebra to allow further computations.

2. Mathematical background. Let $K$ be a field. We denote by

$$
E=K\left\langle e_{1}, \ldots, e_{n}\right\rangle
$$

the exterior algebra of a $K$-vector space $V$ with basis $e_{1}, \ldots, e_{n}$. For any subset $\sigma=\left\{i_{1}, \ldots, i_{d}\right\}$ of $\{1, \ldots, n\}$, with $i_{1}<i_{2}<\cdots<i_{d}$, we write $e_{\sigma}=e_{i_{1}} \wedge \cdots \wedge e_{i_{d}}$, and call $e_{\sigma}$ a monomial of degree $d$. We set $e_{\sigma}=1$, if $\sigma=\varnothing$. The set of monomials in $E$ forms a $K$-basis of $E$ of cardinality $2^{n}$.

In order to simplify the notation, we write $f g=f \wedge g$ for any two elements $f$ and $g$ in $E$. An element $f \in E$ is called homogeneous of degree $j$ if $f \in E_{j}$, where $E_{j}=\bigwedge^{j} V$. An ideal $I$ is called graded if $I$ is generated by homogeneous elements. If $I$ is graded, then $I=\oplus_{j \geq 0} I_{j}$, where $I_{j}$ is the $K$-vector space of all homogeneous elements $f \in I$ of degree $j$. We denote by indeg $(I)$ the initial degree of $I$, i.e., the least degree of a homogeneous generator of $I$.

If $I$ is a graded ideal in $E$, then the function $H_{I}: \mathbb{N} \rightarrow \mathbb{N}$ given by $H_{I}(d)=$ $\operatorname{dim}_{K} I_{d}(i \geq 0)$ is called the Hilbert function of $I$.

Now let $e_{\sigma}=e_{i_{1}} \cdots e_{i_{d}} \neq 1$ be a monomial in $E$. We define

$$
\operatorname{supp}\left(e_{\sigma}\right)=\sigma=\left\{j: e_{j} \operatorname{divides} e_{\sigma}\right\}, \quad \operatorname{m}\left(e_{\sigma}\right)=\max \left\{i: i \in \operatorname{supp}\left(e_{\sigma}\right)\right\} .
$$

Moreover, we set $\mathrm{m}\left(e_{\sigma}\right)=0$ if $e_{\sigma}=1$.

If $M$ is a set of monomials of degree $d<n$ of $E$, the set of monomials of degree $d+1$,

$$
\operatorname{Shad}(M)=\left\{(-1)^{\alpha(\sigma, j)} e_{j} e_{\sigma}: e_{\sigma} \in M, j \notin \operatorname{supp}\left(e_{\sigma}\right), \quad j=1, \ldots, n\right\},
$$

where $\alpha(\sigma, j)=|\{r \in \sigma: r<j\}|$, is called the shadow of $M$ and is denoted by $\operatorname{Shad}(M)$ [Crupi and Ferró 2015, Definition 2.4].

Definition 2.1. Let $I$ be a monomial ideal of $E$. $I$ is called stable if for each monomial $e_{\sigma} \in I$ and each $j<\mathrm{m}\left(e_{\sigma}\right)$ one has $e_{j} e_{\sigma \backslash\left\{\mathrm{m}\left(e_{\sigma}\right)\right\}} \in I$. I is called strongly stable if for each monomial $e_{\sigma} \in I$ and each $j \in \sigma$ one has $e_{i} e_{\sigma \backslash\{j\}} \in I$, for all $i<j$.

If $I$ is a monomial ideal of $E$, we denote by $G(I)$ the unique minimal set of monomial generators of $I$.

Remark 2.2. One can observe that the defining property of a strongly stable ideal needs to be checked only for the set of monomial generators of a monomial ideal. Indeed, let $I$ be a monomial ideal and suppose that for all $e_{\sigma} \in G(I)$, and for all integers $1 \leq i<j \leq n$ such that $j \in \sigma$, one has $e_{i} e_{\sigma \backslash\{j\}} \in I$. Then $I$ is strongly stable. 
Let $e_{\tau} \in I$ be a monomial and $1 \leq i<j \leq n$ be integers such that $j \in \tau$. There exist $e_{\sigma} \in G(I)$ and a monomial $e_{\mu} \in E$ such that $e_{\tau}=e_{\sigma} e_{\mu}$ in $E$.

We distinguish two cases: $j \in \sigma, j \in \mu$. If $j \in \sigma$, then $e_{i} e_{\sigma \backslash\{j\}} \in I$ by assumption, and so $e_{i} e_{\tau \backslash\{j\}}=e_{i} e_{\sigma \backslash\{j\}} e_{\mu} \in I$.

If $j \in \mu$, then $e_{i} e_{\tau \backslash\{j\}}=e_{i} e_{\sigma} e_{\mu \backslash\{j\}} \in I$.

Another class of monomial ideals which plays a relevant role in combinatorial commutative algebra is the class of lexsegment ideals. The lexsegment ideals provide an upper bound for the graded Betti numbers of graded ideals with given Hilbert function [Aramova et al. 1997, Theorem 4.4].

Let $\operatorname{Mon}_{d}(E)$ be the set of all monomials of degree $d \geq 1$ in $E$. Denote by $>_{\text {lex }}$ the lexicographic order on $\operatorname{Mon}_{d}(E)$, i.e., if $e_{\sigma}=e_{i_{1}} e_{i_{2}} \cdots e_{i_{d}}$ and $e_{\tau}=e_{j_{1}} e_{j_{2}} \cdots e_{j_{d}}$ are monomials belonging to $\operatorname{Mon}_{d}(E)$, with $1 \leq i_{1}<i_{2}<\cdots<i_{d} \leq n$ and $1 \leq j_{1}<j_{2}<\cdots<j_{d} \leq n$, then $e_{\sigma}>_{\text {lex }} e_{\tau}$ if $i_{1}=j_{1}, \ldots, i_{s-1}=j_{s-1}$ and $i_{s}<j_{s}$ for some $1 \leq s \leq d$.

Definition 2.3. A nonempty subset $M$ of $\operatorname{Mon}_{d}(E)$ is called a lexsegment of degree $d$ if for all $v \in M$ and all $u \in \operatorname{Mon}_{d}(E)$ such that $u>_{\text {lex }} v$, we have that $u \in M$.

Definition 2.4. A monomial ideal $I$ of $E$ is called a lexsegment ideal (lex ideal, for short) if for all monomials $u \in I$ and all monomials $v \in E$ with $\operatorname{deg} u=\operatorname{deg} v$ and $v>_{\text {lex }} u$, we have $v \in I$.

Equivalently, a monomial ideal $I$ in $E$ is called a lex ideal if $\operatorname{Mon}_{d}(I)$ is a lexsegment for all $d ; \operatorname{Mon}_{d}(I)$ is the set of all monomials of degree $d$ in $I$.

Remark 2.5. Every lex ideal of $E$ is obviously a strongly stable ideal, and consequently a stable ideal.

Now let $a$ and $i$ be two positive integers. Then $a$ has the unique $i$-th Macaulay expansion [Herzog and Hibi 2011, Lemma 6.3.4]

$$
a=\left(\begin{array}{c}
a_{i} \\
i
\end{array}\right)+\left(\begin{array}{c}
a_{i-1} \\
i-1
\end{array}\right)+\cdots+\left(\begin{array}{c}
a_{j} \\
j
\end{array}\right)
$$

with $a_{i}>a_{i-1}>\cdots a_{j} \geq j \geq 1$. We define

$$
a^{(i)}=\left(\begin{array}{c}
a_{i} \\
i+1
\end{array}\right)+\left(\begin{array}{c}
a_{i-1} \\
i
\end{array}\right)+\cdots+\left(\begin{array}{c}
a_{j} \\
j+1
\end{array}\right) .
$$

We also set $0^{(i)}=0$ for all $i \geq 1$.

The next theorem describes the possible Hilbert functions of graded $K$-algebras of the form $E / I$, with $I$ a graded ideal in $E$. It is the precise analogue to Macaulay's theorem [Bruns and Herzog 1993; Eisenbud 1995] which describes the possible Hilbert functions of standard graded $K$-algebras. 
Theorem 2.6 [Aramova et al. 1997, Theorem 4.1]. Let $\left(h_{1}, \ldots, h_{n}\right)$ be a sequence of nonnegative integers. Then the following conditions are equivalent:

(a) $1+\sum_{i=1}^{n} h_{i} t^{i}$ is the Hilbert series of a graded $K$-algebra $E / I$.

(b) $0<h_{i+1} \leq h_{i}^{(i)}, 0<i \leq n-1$.

Theorem 2.6 is known as the Kruskal-Katona theorem. Its proof points out that if $I$ is a graded ideal of $E$, then there exists a unique lex ideal of $E$, usually denoted by $I^{\text {lex }}$, such that $H_{E / I}=H_{E / I^{\text {lex }}}$.

More precisely, if $\left(1, h_{1}, \ldots, h_{n}\right)$ is a sequence of nonnegative integers such that

(i) $h_{1} \leq n$,

(ii) $0<h_{i+1} \leq h_{i}^{(i)}, 0<i \leq n-1$,

then there exists a unique lex ideal $J$ (indeg $J \geq 1$ ) of an exterior algebra $E$ with $n$ generators over a field $K$ such that $H_{E / J}(d)=h_{d}(d=0, \ldots, n)$.

If $1+\sum_{i=1}^{n} h_{i} t^{i}$ is the Hilbert series of a graded $K$-algebra $E / I$, then the sequence $\left(1, h_{1}, \ldots, h_{n}\right)$ is called the Hilbert sequence of $E / I$.

From the Kruskal-Katona theorem, one can deduce that a sequence of nonnegative integers $\left(h_{0}, h_{1}, \ldots, h_{n}\right)$ is the Hilbert sequence of a graded $K$-algebra $E / I$, with $I \subsetneq E$ a graded ideal of initial degree $\geq 1$, if $h_{0}=1$, and (i) and (ii) hold.

From now on, when we speak about Hilbert sequences we refer to Hilbert sequences of quotients of an exterior algebra.

3. EXAMPLES. In this section, we collect some examples in order to describe the algorithms. Our implementation works in any characteristic.

Example 3.1. Given a monomial ideal $I$ in an exterior algebra $E$, we illustrate how some functions from our package allow one to check whether $I$ is stable, strongly stable, or lex, and to produce stable or strongly stable ideals containing $I$. The core of the algorithms is based on the fact that the minimal monomial generators of a stable or strongly stable ideal must satisfy the criterion in Definition 2.1 (see Remark 2.2) and on the fact that the shadow of a lexsegment of monomials is again a lexsegment [Herzog and Hibi 2011].

Macaulay2, version 1.10

with packages: ConwayPolynomials, Elimination, Integralclosure, InverseSystems, LLLBases, PrimaryDecomposition, ReesAlgebra, TangentCone

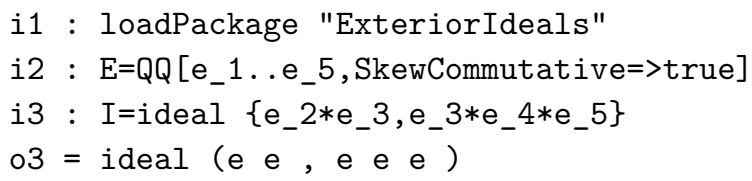




\section{o3 : Ideal of $\mathrm{E}$ \\ i4 : isStableIdeal I \\ 04 = false}

The ideal $I$ is not stable. Indeed, the monomial $e_{1} e_{2}$ is not in $I$ even though $e_{2} e_{3}$ is. Hence, by the function stableIdeal (ideal), we compute the smallest stable ideal $(I s)$ containing $I$ :

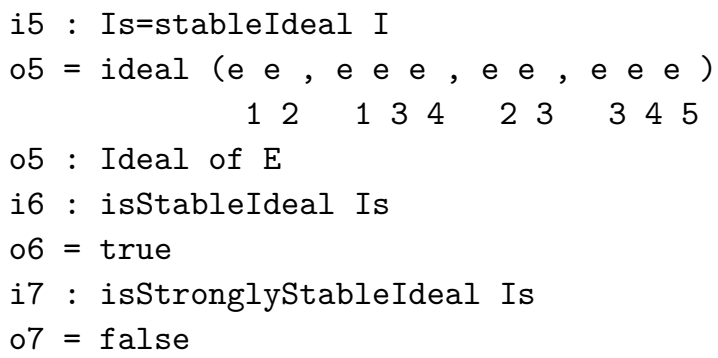

The ideal $I s$ is stable but not strongly stable in $E$. Note that the monomial $e_{1} e_{3}$ is not in $I s$ even though $e_{2} e_{3}$ is.

Using the function stronglyStableIdeal (ideal), we compute the smallest strongly stable ideal (Iss) containing $I s$, and consequently $I$ :

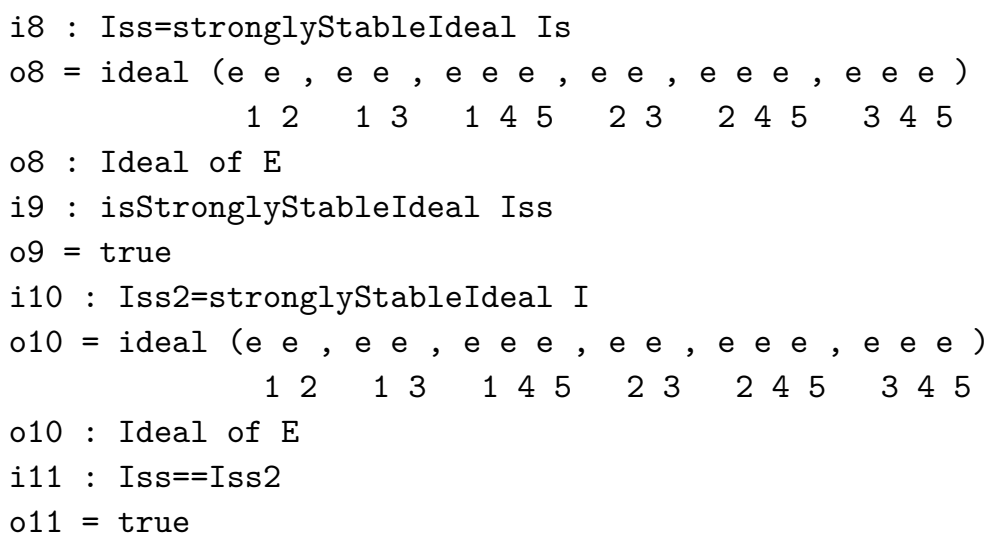

The ideal $I s s$ is not a lex ideal in $E$. Indeed, the monomial $e_{1} e_{4}$ does not belong to $I s s$, but $e_{1} e_{4}>$ lex $e_{2} e_{3}$. One can verify this by the function isLexIdeal (ideal):

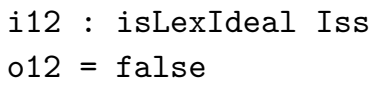

Example 3.2. Letting $E$ be an exterior algebra with $n$ generators over a field $K$ and $h=\left(h_{0}, h_{1}, \ldots, h_{n}\right)$ be a sequence of nonnegative integers, we describe how one can verify if $h$ is a Hilbert sequence.

The key tools in our algorithm are the functions isHilbertSequence (list, exterior algebra) and lexIdeal (list, exterior algebra). The first function verifies if a list of nonnegative integers of length $n+1$ is a Hilbert function; the 
second one returns a lex ideal of $E$ if and only if the list is a Hilbert sequence. In more detail, if $\left(h_{0}, h_{1}, \ldots, h_{n}\right)$ is a Hilbert sequence, the lex ideal of $E$ produced by the function lexIdeal $\left(\left\{h_{0}, \ldots, h_{n}\right\}, E\right)$ is the unique lex ideal $I$ of $E$ with $H_{E / I}(d)=h_{d}(d=0, \ldots, n)$. The procedure for the computation of the required lex ideal is based on the constructive proof of Theorem 2.6 (see [Aramova et al. 1997, Theorem 4.1, (b) $\Rightarrow$ (a)]).

We start with some examples of sequences which are not Hilbert sequences. The property is verified by using either isHilbertSequence(list, exterior algebra) or lexIdeal (list, exterior algebra):

Macaulay2, version 1.10

with packages: ConwayPolynomials, Elimination, IntegralClosure, InverseSystems, LLLBases, PrimaryDecomposition, ReesAlgebra, TangentCone

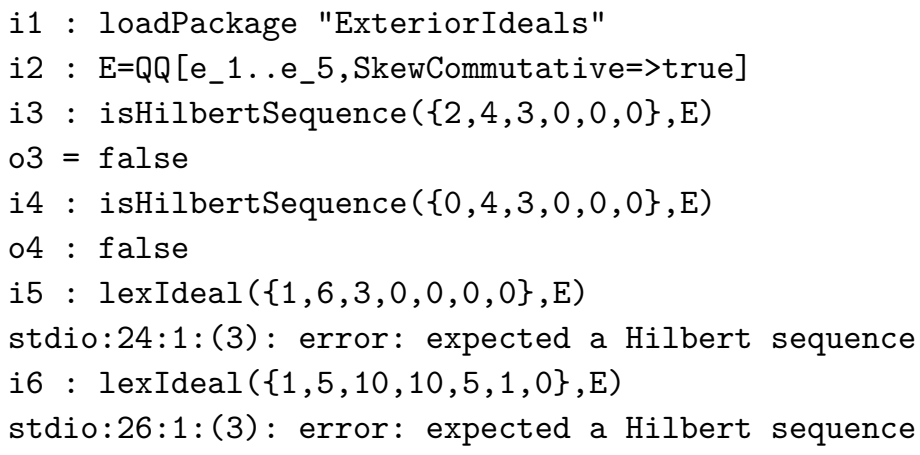

Moreover, the next statements provide some examples of the lex ideal produced by a Hilbert sequence. The length of the sequence can be at most $n+1$; if the length is less than $n+1$, then the sequence will be completed by adding zeros on the right.

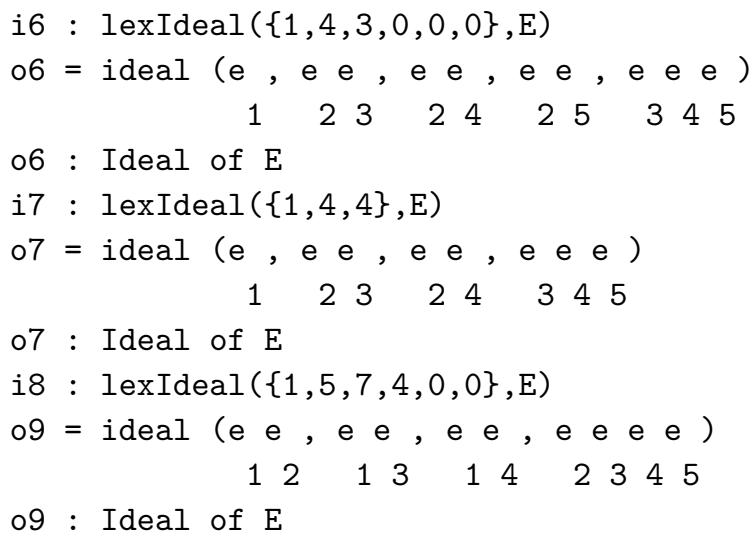

The function lexIdeal (list, exterior algebra), defined above, also plays a relevant role in the next algorithm. 
Example 3.3. Given an exterior algebra $E$ and a graded ideal $I$ in $E$, we illustrate how to obtain the unique lex ideal $I^{\text {lex }}$ with the same Hilbert function as $I$. In more detail, we describe two different methods for computing such a lex ideal.

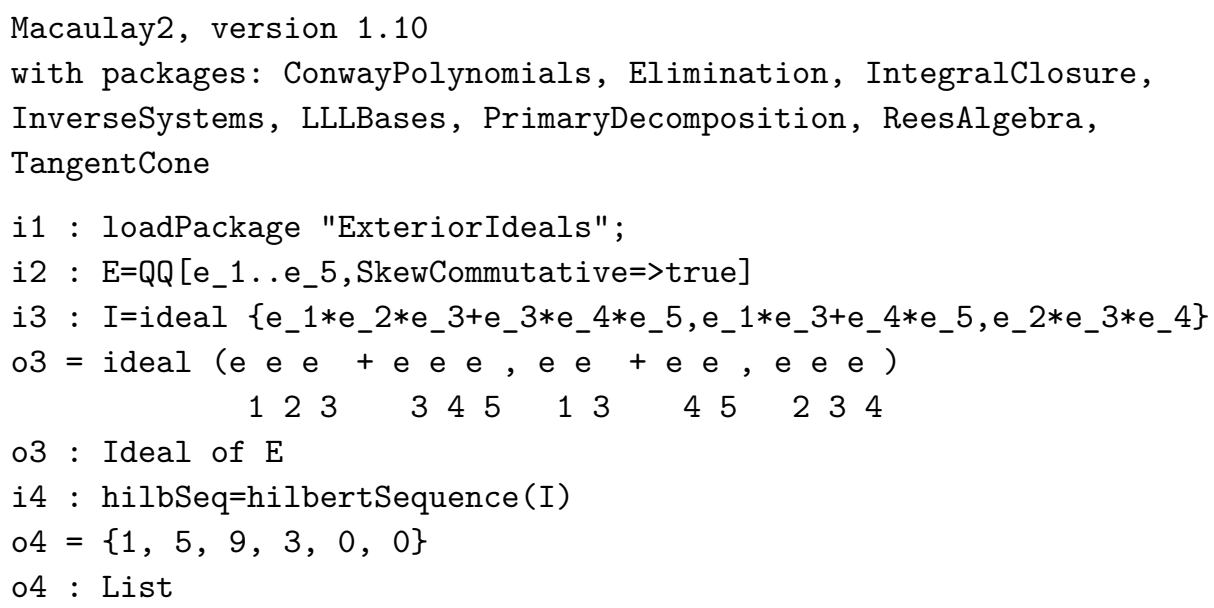

A first way for computing the lex ideal we are looking for is to use the function lexIdeal (list, exterior algebra):

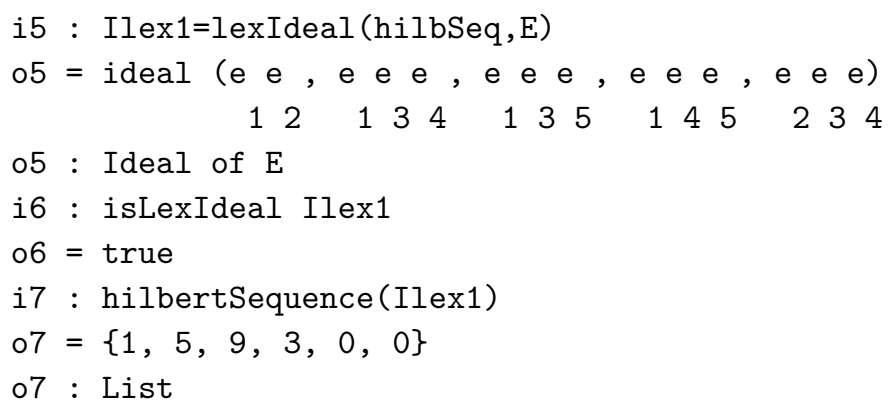

and a second one is via the new function lexIdeal (ideal), which returns directly the required lex ideal:

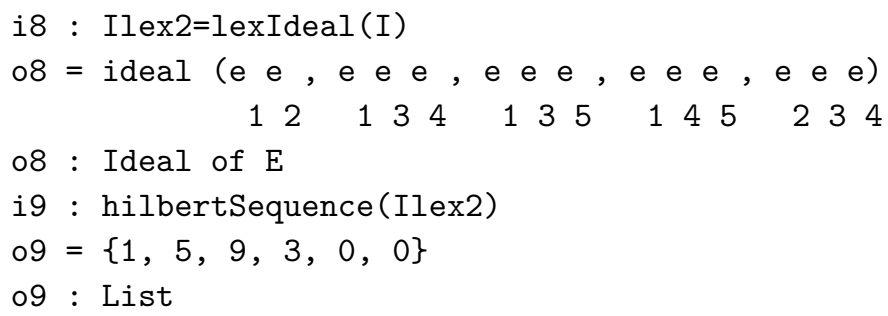

Finally, our last example is related to the algorithm for the computation of Hilbert sequences.

Example 3.4. Given an exterior algebra $E$, we illustrate how to get all the Hilbert sequences of quotients of $E$. 
Macaulay2, version 1.10

with packages: ConwayPolynomials, Elimination, IntegralClosure, InverseSystems, LLLBases, PrimaryDecomposition, ReesAlgebra, TangentCone

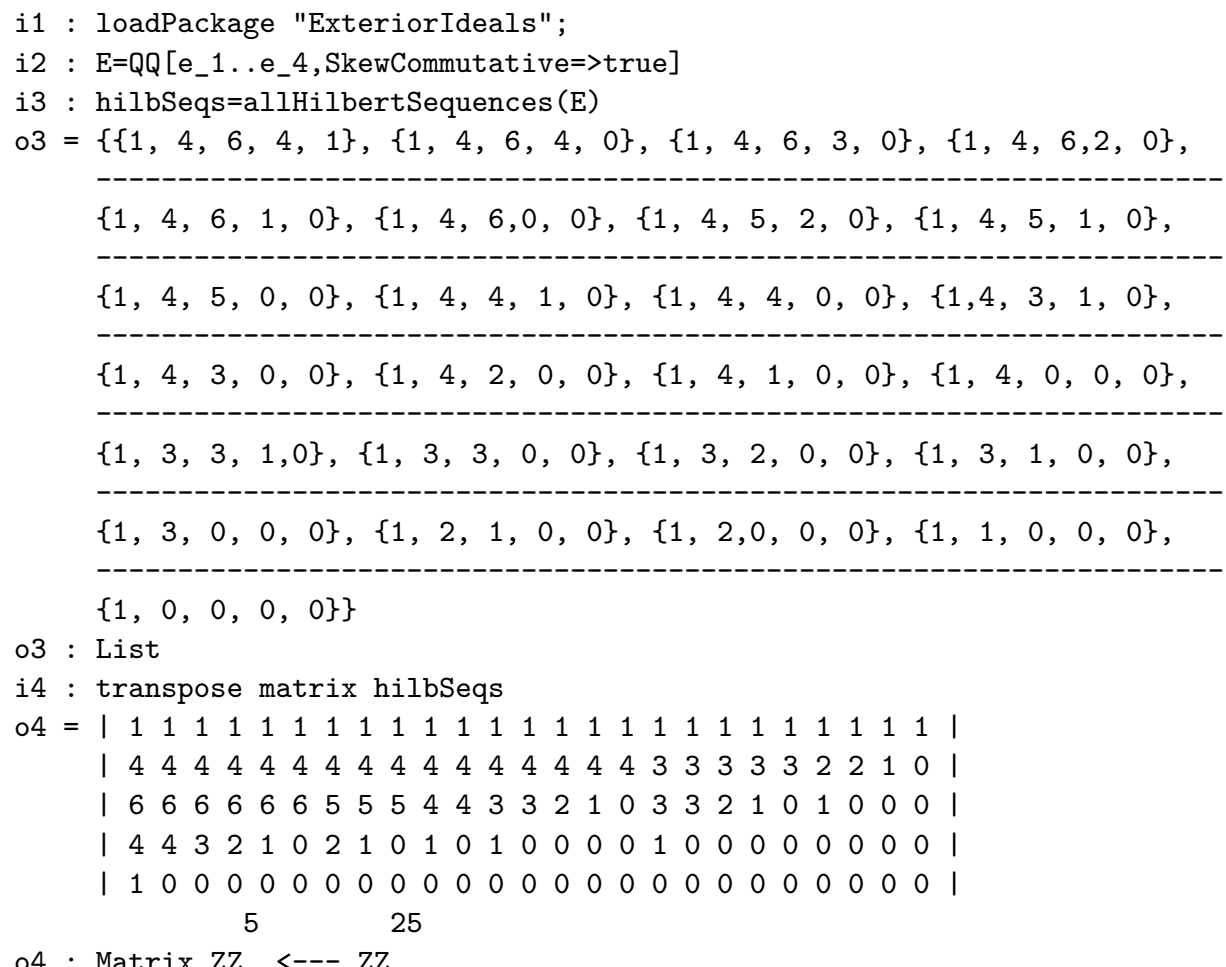

Matrix ZZ <--- ZZ

Note that the method allHilbertSequences returns an object of type List; for a more compact view it could be displayed as a matrix.

4. CONClusions And PeRspectives. The algorithms described in the examples above are part of a Macaulay2 package ExteriorIdeals.m2, which has been tested with Macaulay2 version 1.10. We are confident that this package may prove useful for further applications. Indeed, to the best of our knowledge, it seems that no packages for manipulating monomial ideals in an exterior algebra have been implemented, though functions for computing monomial ideals in a polynomial ring are available in many computer algebra systems (for instance, [CoCoA], [Macaulay2] and [Singular]).

We believe it would be nice to implement such a package for monomial modules over an exterior algebra. This task is currently under investigation by the authors.

ACKNOWLEDGEMENTS. The authors thank the anonymous referees for their useful comments that improved the quality of the paper.

SUPPLEMENT. The online supplement contains version 1.0 of ExteriorIdeals .m2. 


\section{REFERENCES.}

[Aramova et al. 1997] A. Aramova, J. Herzog, and T. Hibi, "Gotzmann theorems for exterior algebras and combinatorics", J. Algebra 191:1 (1997), 174-211. MR Zbl

[Aramova et al. 2000] A. Aramova, L. L. Avramov, and J. Herzog, "Resolutions of monomial ideals and cohomology over exterior algebras", Trans. Amer. Math. Soc. 352:2 (2000), 579-594. MR Zbl

[Bruns and Herzog 1993] W. Bruns and J. Herzog, Cohen-Macaulay rings, Cambridge Studies in Advanced Mathematics 39, Cambridge University Press, 1993. MR Zbl

[CoCoA] J. Abbott, A. M. Bigatti, and L. Robbiano, "CoCoA: a system for doing Computations in Commutative Algebra", available at http://cocoa.dima.unige.it.

[Crupi 2015] M. Crupi, "Algebraic invariants of graded ideals with a given Hilbert function in an exterior algebra”, Bull. Math. Soc. Sci. Math. Roumanie (N.S.) 58(106):4 (2015), 393-403. MR $\mathrm{Zbl}$

[Crupi and Ferró 2015] M. Crupi and C. Ferró, "Bounding Betti numbers of monomial ideals in the exterior algebra", Pure Appl. Math. Q. 11:2 (2015), 267-281. MR Zbl

[Crupi and Utano 1999] M. Crupi and R. Utano, "Upper bounds for the Betti numbers of graded ideals of a given length in the exterior algebra", Comm. Algebra 27:9 (1999), 4607-4631. MR Zbl

[Crupi and Utano 2007] M. Crupi and R. Utano, "Classes of graded ideals with given data in the exterior algebra", Comm. Algebra 35:8 (2007), 2386-2408. MR Zbl

[Eisenbud 1995] D. Eisenbud, Commutative algebra with a view toward algebraic geometry, Graduate Texts in Mathematics 150, Springer, 1995. MR Zbl

[Eisenbud et al. 2003] D. Eisenbud, S. Popescu, and S. Yuzvinsky, "Hyperplane arrangement cohomology and monomials in the exterior algebra", Trans. Amer. Math. Soc. 355:11 (2003), 4365-4383. MR Zbl

[Gasharov 1997] V. Gasharov, "Extremal properties of Hilbert functions", Illinois J. Math. 41:4 (1997), 612-629. MR Zbl

[Herzog and Hibi 2011] J. Herzog and T. Hibi, Monomial ideals, Graduate Texts in Mathematics 260, Springer, 2011. MR Zbl

[Macaulay2] D. R. Grayson and M. E. Stillman, "Macaulay2: a software system for research in algebraic geometry", available at http://www.math.uiuc.edu/Macaulay2.

[Murai 2011] S. Murai, "Free resolutions of lex-ideals over a Koszul toric ring", Trans. Amer. Math. Soc. 363:2 (2011), 857-885. MR Zbl

[Shakin 2004] D. A. Shakin, "Hilbert functions and Betti numbers of homogeneous ideals in an exterior algebra", Uspekhi Mat. Nauk 59:5(359) (2004), 165-166. MR Zbl

[Shakin 2005] D. A. Shakin, "Piecewise-lexsegment ideals in exterior algebras", Mat. Sb. 196:2 (2005), 287-307. MR Zbl

[Singular] W. Decker, G.-M. Greuel, G. Pfister, and H. Schönemann, "SInGUlaR 4-1-1 - A computer algebra system for polynomial computations", available at http://www.singular.uni-kl.de.

Received: 28 Aug 2017 Revised: 15 May 2018 ACCEPted: 24 Jun 2018

LUCA AMATA:

lamata@unime.it

Department of Mathematics and Computer Sciences, Physics and Geological Sciences, University of Messina, Messina, Italy

MARILENA CRUPI:

mcrupi@unime.it

Department of Mathematics and Computer Sciences, Physics and Geological Sciences, University of Messina, Messina, Italy 

HeLP: a GAP package for torsion units in integral group rings

Andreas Bächle and Leo Margolis

A software package to compute automorphisms of graded algebras

Simon Keicher

A package for computations with classical resultants

Giovanni Staglianò

The Space Curves package in Macaulay2

Mengyuan Zhang

The ReesAlgebra package in Macaulay2

David Eisenbud

A Macaulay2 package for computations with rational maps

Giovanni Staglianò

ExteriorIdeals: a package for computing monomial ideals in an exterior algebra

Luca Amata and Marilena Crupi

Software for computing conformal block divisors on $\bar{M}_{0, n}$

David Swinarski

Divisor Package for Macaulay2

Karl Schwede and Zhaoning Yang 